\title{
Os desafios administrativos na adoção de sistemas de controle para empresas globalizadas
}

\begin{abstract}
Robson Augusto Dainez Condé Mestrado em andamento em Ciências Contábeis pela Universidade do Estado do Rio de Janeiro - UERJ Auditor Fiscal da Fazenda do Estado do Espírito Santo Rua São Francisco Xavier, 524, $9^{\circ}$ andar, Bloco E. Maracanã. Rio de Janeiro/RJ. CEP: 20550-013 E-mail: robsonconde.mcc.uerj@hotmail.com

Renato Santiago Quintal Mestrado em andamento em Ciências Contábeis pela Universidade do Estado do Rio de Janeiro - UERJ Oficial da Ativa do Corpo de Intendentes da Marinha do Brasil Rua São Francisco Xavier, 524, $9^{\circ}$ andar, Bloco E. Maracanã. Rio de Janeiro/RJ. CEP: 20550-013

E-mail: rsantiago79@hotmail.com

Leonardo Portugal Barcellos Mestrado em andamento em Ciências Contábeis pela Universidade do Estado do Rio de Janeiro - UERJ Analista do Ministério do Planejamento, Orçamento e Gestão - MPOG Rua São Francisco Xavier, 524, $9^{\circ}$ andar, Bloco E. Maracanã. Rio de Janeiro/RJ. CEP: 20550-013

E-mail: Ipbarcellos@gmail.com

Josir Simeone Gomes Doutorado em Administração pela Universidade Federal do Rio de Janeiro -UFRJ Pós-Doutorado na Universidade Carlos III de Madrid Professor da Universidade a Grande Rio - UNIGRANRIO Rua da Lapa, 86. Centro. Rio de Janeiro/RJ. CEP: 25318-804 E-mail: josirgomes@superig.com.br
\end{abstract}

\section{RESUMO}

O artigo objetiva apresentar a evolução dos estudos sobre internacionalização, bem como os entraves administrativos enfrentados pelas organizações na adaptação de seus sistemas de controle gerencial para atender as características de uma empresa globalizada. Foi empreendida uma revisão bibliográfica a fim de evidenciar a importância de estudos na área de controle gerencial. Os resultados apontam que a decisão de uma empresa em ampliar suas operações para outros países deve estar amparada em um estudo aprofundado sobre as implicações práticas desta decisão. 
Os desafios administrativos na adoção de sistemas de controle para empresas globalizadas

Robson Augusto Dainez Condé, Renato Santiago Quintal, Leonardo Portugal Barcellos, Josir Simeone

Gomes

Adicionalmente, o seu sistema de controle gerencial deve ser modificado, buscando retratar uma nova estratégia da unidade de negócios.

Palavras-chave: Internacionalização. Globalização. Sistemas de Controle Gerencial.

\section{Administrative challenges in the adoption of control systems to globalized companies}

\section{ABSTRACT}

The article present the evolution of studies on internationalization, as well as the administrative barriers organizations faces in adapting its Managerial Control systems to meet the characteristics of a globalised company. A literature review was undertaken in order to highlight the importance of studies in the area of managerial control. The results suggest that a company's decision to expand its operations to other countries must be based on an in-depth study on the practical implications of this decision. Additionally, his managerial control system must be modified, seeking to portray a new business unit strategy.

Keywords: Internationalization. Globalization. Management Control Systems.

\section{INTRODUÇÃO}

A globalização da economia tem possibilitado que bens e serviços sejam negociados transnacionalmente. Nesse contexto, as empresas enfrentam forte concorrência e, diante disso, devem estabelecer metas que levem em consideração aspectos típicos de uma estratégia de dimensão internacional (GOMES; OLIVEIRA, 2011).

Como exemplo pode se citar que uma empresa pode projetar seu produto na Europa, com componentes fabricados na China e montados nos EUA, e pode revendêlos em diversos países da América do Sul. Os preços dos itens e da montagem do produto podem ser determinados pelo estudo do menor custo a ser agregado ao produto. Além disso, o financiamento deste investimento pode ser oriundo do mercado europeu e as transações podem ser efetuadas em diversas moedas, com 
Os desafios administrativos na adoção de sistemas de controle para empresas globalizadas

Robson Augusto Dainez Condé, Renato Santiago Quintal, Leonardo Portugal Barcellos, Josir Simeone

Gomes

possibilidades de lucros e perdas característicos dos contratos de câmbio internacionais, com cobertura a termo.

Essa intrincada dinâmica gera um complexo problema para os sistemas de controle gerencial, pois surgem, nesse contexto, questões relacionadas à alocação dos resultados dessas entidades globais e também relacionadas ao estabelecimento e monitoramento de metas. As abordagens de controle nessas empresas precisam ser suficientes para lidar com diferentes conjunturas político-econômicas, culturais, padrões de consumo, moeda, língua, entre outros. Desse modo, uma vez estabelecidas metas e responsabilidades pelo resultado, devem ser capazes de comparar o planejado com o que foi de fato alcançado, adotando as medidas corretivas necessárias, mantendo, assim, a lógica de controle gerencial.

Hodiernamente, dadas as constantes modificações decorrentes da globalização, há uma crescente necessidade de se adotar um novo modelo de controle gerencial, pois aquilo que era considerado apropriado para uma economia estável deve, agora, ser substituído por uma visão de controle gerencial bem diferente. A literatura sobre o tema e a prática das companhias indica que, ao contrário de uma empresa multinacional, uma empresa globalizada deve ser administrada de forma centralizada e precisa de mecanismos de controle que mensurem e recompensem o desempenho baseado no cumprimento de seus objetivos estratégicos.

Desta forma, o problema central deste estudo pode ser assim formulado: Qual a importância do processo de internacionalização das empresas e os cuidados que a administração deve ter na adaptação dos sistemas de controle gerencial (SCG) para empresas globalizadas? Diante desta questão, o objetivo do estudo é apresentar uma revisão da literatura sobre internacionalização de empresas de modo a apontar as melhores práticas na utilização de SCG em empresas globalizadas.

Gomes e Oliveira (2011), considerando a quantidade de trabalhos publicados sobre internacionalização de empresas no âmbito do Programa de Mestrado em Ciências Contábeis da UERJ, constatam um significativo aumento no volume de pesquisas nesta área a partir de 2006. Corroborando a relevância do tema, Fernandes (2006) reforça a importância da globalização para empresas brasileiras, estabelecendo 
Os desafios administrativos na adoção de sistemas de controle para empresas globalizadas

Robson Augusto Dainez Condé, Renato Santiago Quintal, Leonardo Portugal Barcellos, Josir Simeone

Gomes

que uma crise econômica pode afetar setores da economia nacional e pode resultar em estagnação ou até mesmo retração desses mercados, além de uma acirrada concorrência de empresas nacionais e estrangeiras. Acrescenta, ainda, que muitos empresários brasileiros estão encontrando na globalização a oportunidade de precaução, mantendo suas empresas rentáveis e obtendo um incremento em seus lucros.

\section{REVISÃO DA LITERATURA}

Gomes e Rodrigues (2005), com base em Bartlett e Ghoshal (1992), Canals (1994) e Dyment (1987), apresentam uma proposta de classificação para empresas internacionalizadas que está sintetizada no Quadro 1.

\begin{tabular}{|l|l|}
\hline \multicolumn{1}{|c|}{ CLASSIFICAÇÃO } & \multicolumn{1}{c|}{ SíNTESE } \\
\hline $\begin{array}{l}\text { Empresa } \\
\text { exportadora }\end{array}$ & $\begin{array}{l}\text { Normalmente as unidades operacionais ficam no país de origem, sendo } \\
\text { considerada uma fase inicial do processo de internacionalização. }\end{array}$ \\
\hline $\begin{array}{l}\text { Empresa } \\
\text { multinacional }\end{array}$ & $\begin{array}{l}\text { A empresa explora suas vantagens competitivas diversificando as } \\
\text { atividades em outros países. O objetivo é a reprodução quase exata da } \\
\text { matriz em cada uma das filiais no exterior; }\end{array}$ \\
\hline Empresa global & $\begin{array}{l}\text { A empresa adota estratégias coordenadas com todos os países onde opera. } \\
\text { Dessa forma, o objetivo é adquirir vantagem competitiva em termos de } \\
\text { custos e receitas através de operações centralizadas em escala global. }\end{array}$ \\
\hline $\begin{array}{l}\text { Empresa } \\
\text { transnacional }\end{array}$ & $\begin{array}{l}\text { A empresa combina uma máxima eficiência econômica, uma máxima } \\
\text { capacidade de responder a mercados locais e uma flexibilidade para } \\
\text { transmitir as experiências que surgem em alguns países para toda a } \\
\text { organização. A empresa transnacional "busca eficiência, não pela eficiência } \\
\text { em si, mas como meio de alcançar competitividade global" (BARTLETT e } \\
\text { GHOSHAL, 1992, p.85). }\end{array}$ \\
\hline
\end{tabular}

Quadro 1: Síntese da classificação proposta por Gomes e Rodrigues (2005)

Fonte: Gomes e Rodrigues (2005, p. 7).

Mazo et al. (2004) apresentam uma ilustração da relação entre as unidades de cada modelo de empresa como consta na Figura 1. 
Os desafios administrativos na adoção de sistemas de controle para empresas globalizadas Robson Augusto Dainez Condé, Renato Santiago Quintal, Leonardo Portugal Barcellos, Josir Simeone

Gomes

Figura 1: Relação entre empresas

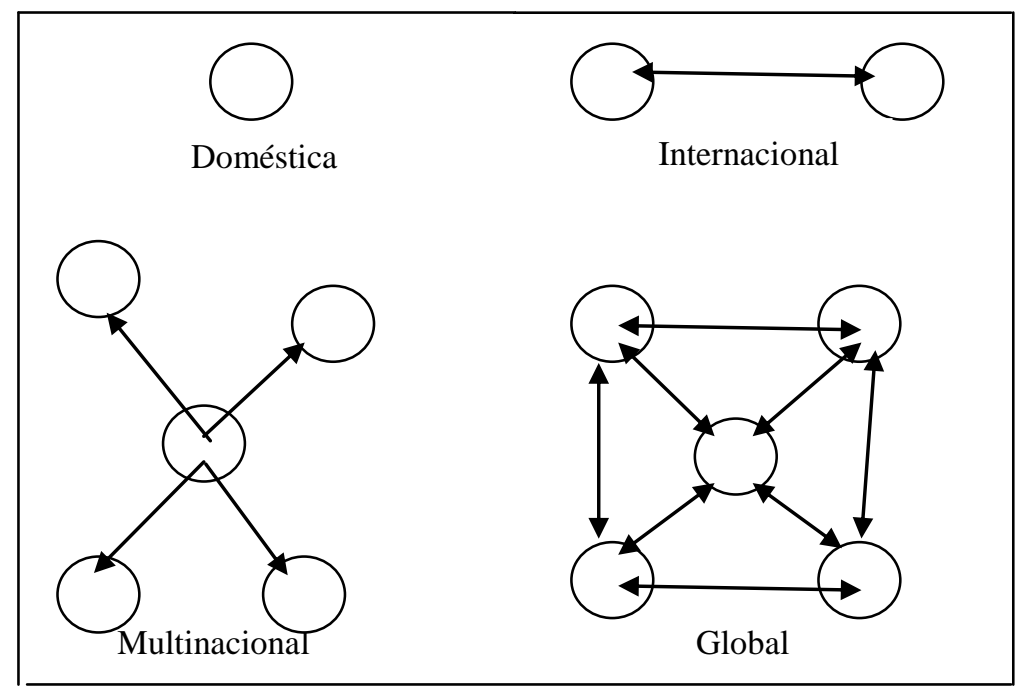

Fonte: Adaptado de Mazo et al., 2004.

As empresas podem se estabelecer como nacionais, multinacionais ou globais e podem migrar de um modelo para outro. Acompanhando esta possível mudança, o sistema de controle gerencial deve ser modificado para retratar a nova estratégia que se faz necessária às unidades de negócios. Se os controles anteriores forem mantidos, as ações da empresa podem ser desviadas de seu foco e ela pode fracassar na tentativa de se tornar globalizada (DYMENT, 1987).

No entendimento de Dyment (1987), empresa globalizada é aquela organização que detém alguma vantagem sobre os demais concorrentes em função de possuir estratégia capaz de envolver todos os países em que atua. Ao contrário dela, as multinacionais adotam estruturas organizacionais e estratégias específicas para cada um dos países.

Por seu turno, Otley (1994) ressalta que nas empresas internacionalizadas as condições de incerteza enfrentadas pelos gestores representam um grande desafio para os mecanismos de controle gerencial. Em tais situações a habilidade gerencial de reagir às frequentes mudanças impostas pelas condições de mercado faz-se cada vez mais necessária.

Merchant (1998) comenta que as organizações multinacionais que realizam operações em vários países, são instituições muito importantes na economia global, 
Os desafios administrativos na adoção de sistemas de controle para empresas globalizadas

Robson Augusto Dainez Condé, Renato Santiago Quintal, Leonardo Portugal Barcellos, Josir Simeone

Gomes

pois os resultados de suas operações representam uma grande parcela de todo o valor negociado no mundo. Nestas instituições, embora a autoridade seja descentralizada em grande parte para as unidades de negócios de cada país, o foco dos sistemas de controle de gestão permanece sobre os resultados financeiros.

No processo de internacionalização, a exportação é considerada por muitos autores como o primeiro passo da empresa na busca por mercados externos. Vencida esta etapa, a organização começará a se familiarizar com a cultura de outros países e, principalmente, com as preferências e necessidades do respectivo mercado consumidor. Assim, absorvidas essas características, a entidade possuirá conhecimento diferencial sobre como satisfazer a parcela de mercado que visa a alcançar (URBASCH, 2004). Nessa linha, Rocha (2004) acrescenta que o idioma, as características do mercado, o sistema político e a distância são algumas das principais dificuldades encontradas na negociação entre dois países.

Anthony e Govindarajan (2002) corroboram esta análise ao sustentar que as diferenças culturais existentes entre os países podem afetar significativamente 0 sistema de controle gerencial da organização, sendo capaz de promover sensíveis alterações sobre sua aplicação. Os autores destacam, ainda, que os preços de transferência e as taxas de câmbio são dois aspectos problemáticos nas operações com o exterior. Mesmo assim, apesar dessas dificuldades, as empresas brasileiras têm conseguido superá-las e já existem diversos casos de organizações que obtiveram sucesso ao ampliar o seu horizonte para além das fronteiras do País.

Rocha, Silva e Carneiro (2007) abordam o contexto histórico desse processo e afirmam que somente a partir da década de 1990 as empresas nacionais deram início ao processo de internacionalização. Destacam, ainda, que esse processo deve ser precedido da análise custo-benefício do investimento, identificando as vantagens que as empresas terão em relação a concorrência e, tais informações devem constituir o principal subsídio para a decisão.

Consoante Dyment (1987), existe pouca diferença entre os sistemas de controles internos adotados pelas corporações multinacionais e aqueles adotados pelas empresas nacionais. Em verdade, o sistema deve ser alterado sempre que se tratar de 
Os desafios administrativos na adoção de sistemas de controle para empresas globalizadas Robson Augusto Dainez Condé, Renato Santiago Quintal, Leonardo Portugal Barcellos, Josir Simeone

Gomes

uma empresa cuja estratégia venha a ser adotada em nível mundial. Enquadram-se nessa previsão as empresas globalizadas, que devem ser gerenciadas de forma bem diferente de empresas nacionais ou multinacionais, pois seus sistemas de controle gerencial devem ser estruturados de modo a atender à estratégia mundial.

Conforme indicado no Gráfico 1, Gomes e Oliveira (2011) identificaram um significativo aumento no volume de pesquisas sobre internacionalização de empresas a partir do ano de 2006. Adicionalmente, foi reconhecida a interação entre professores e alunos, sinergia usada para a alavancagem da produção científica em torno do tema.

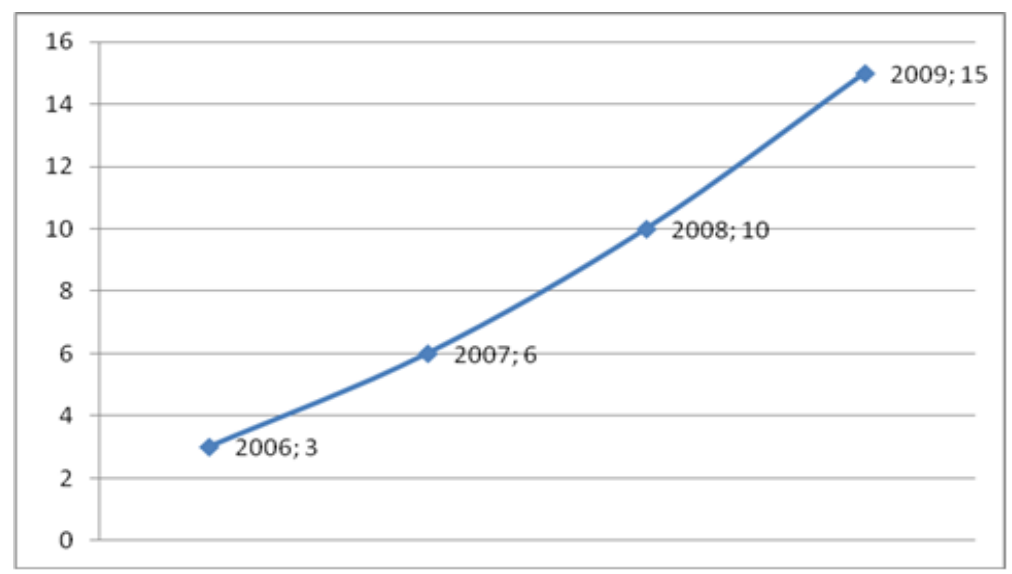

Gráfico 1 - Pesquisas sobre o tema internacionalização produzidas pelo Mestrado em Ciências Contábeis da UERJ -2006 a 2009

Fonte: Gomes e Oliveira (2011, p. 9)

\subsection{Sistemas de Controle Gerencial}

Dyment (1987) estabelece que para uma empresa nacional, o sistema de controle gerencial deve consistir de: (a) sistemas de custos-padrão que possam ser comparados com os custos reais; (b) orçamentos mensais e relatórios de despesas por setor de responsabilidade organizacional; (c) planejamento de longo prazo orientado para a aplicação dos recursos; e (d) planejamento estratégico que compreenda todas as atividades de gerenciamento.

Por outro lado, de acordo com Merchant (1998), em empresas multinacionais a autoridade deve ser descentralizada para as unidades de negócios de cada país e os 
Os desafios administrativos na adoção de sistemas de controle para empresas globalizadas

Robson Augusto Dainez Condé, Renato Santiago Quintal, Leonardo Portugal Barcellos, Josir Simeone

Gomes

sistemas de controle de gestão devem estar focados nos resultados financeiros. Dyment (1987) acrescenta que, para essas empresas, os sistemas devem ser alterados para atender às exigências legais e gerenciais das operações em cada país, considerando: (a) relatórios fiscais e tributários exigidos em cada país; e (b) orçamentos nacionais e planos plurianuais comparados aos resultados, cujos documentos devem ser enviados para as sedes regionais e internacionais da empresa para análise e consolidação.

Apesar da diversidade do mercado, das condições tecnológicas de cada país e dos preços de transferência, que normalmente não podem ser controlados pelos executivos, os relatórios dos resultados de cada país são importantíssimos para os executivos, cujo trabalho depende da arte de prever os resultados de suas ações. Apesar de Anthony e Govindarajan (2002) alertarem que os preços de transferência e as taxas de câmbio constituem dois aspectos problemáticos nas operações com o exterior, as empresas brasileiras têm conseguido superar essas dificuldades.

Os relatórios de controle gerencial de uma multinacional devem utilizar um sistema-padrão mundial de classificação contábil, projetado, inicialmente, para facilitar a consolidação dos resultados da matriz da corporação. Se a matriz for sediada nos Estados Unidos, por exemplo, a consolidação fornecerá relatórios para a SEC, para acionistas e para a Receita Federal (DYMENT, 1987).

Nas empresas multinacionais, o trabalho dos contadores deve atender à seguinte ordem de prioridade: primeiramente, a elaboração dos relatórios para a matriz; em seguida, os relatórios para os órgãos fiscais e tributários locais; e por último, as informações para auxiliar a administração local a controlar suas ações e aperfeiçoar a sua posição estratégica em relação à concorrência local.

Dyment (1987) afirma que os sistemas de informação gerencial das empresas multinacionais podem ser aperfeiçoados por meio da concepção de processo capaz de identificar inconsistências nos objetivos dos relatórios preparados para os acionistas, para os órgãos tributários e para a gerência. 
Os desafios administrativos na adoção de sistemas de controle para empresas globalizadas

Robson Augusto Dainez Condé, Renato Santiago Quintal, Leonardo Portugal Barcellos, Josir Simeone

Gomes

\section{PROCESSO DE GLOBALIZAÇÃO DAS EMPRESAS}

As empresas globalizadas possuem exigências bem distintas das multinacionais. Nas empresas globalizadas a estratégia deve atravessar fronteiras, enquanto nas multinacionais as estratégias dos gerentes focalizam a situação local, os competidores locais e a economia local. As decisões do executivo da empresa globalizada envolvem, em uma estratégia mundial integrada, todos os aspectos da cadeia de valor adicionados ao produto, desde a pesquisa e desenvolvimento, passando pela fabricação e alcançando a distribuição do produto. (DYMENT, 1987)

Como consequência do fenômeno conhecido por globalização, pessoas de várias partes do mundo passaram a desejar produtos similares. Tal convergência de gostos e comportamentos dos consumidores tem acarretado um constante processo de homogeneização da demanda mundial, sendo que a padronização desta demanda passou a ser estratificada, principalmente, segundo o poder aquisitivo do consumidor, o que varia consideravelmente de acordo com o país em que atua. A título de exemplo, um produto muito demandado pela classe média americana pode ser um produto de classe alta na Índia e, simultaneamente, um produto sem mercado em muitos países do continente africano.

Nesse contexto, Dyment (1987) relata que uma empresa globalizada, como forma de obter maior vantagem estratégica, pode selecionar a localização em que vai produzir um os componentes de determinado produto, bem como a abordagem gerencial que será utilizada em cada um de seus itens produzidos. Por exemplo:

- A matéria-prima pode ser adquirida em locais que possuam técnicas de pronto reabastecimento do estoque;

- A produção automatizada pode ser implantada onde se dispõe de mão-deobra especializada;

- A produção pelo trabalho intensivo pode ser adotada nos locais com baixos custos salariais;

- O transporte pode ser localizado onde os custos sejam relativamente baixos; 
Os desafios administrativos na adoção de sistemas de controle para empresas globalizadas Robson Augusto Dainez Condé, Renato Santiago Quintal, Leonardo Portugal Barcellos, Josir Simeone

- A mesma equipe de vendas pode atuar em diversos países, sabendo-se que a utilização de técnicas apropriadas de venda reduzirá os custos.

$\mathrm{O}$ autor aduz que a homogeneização da demanda e a consequente adoção de técnicas de padronização da produção mundial permite considerável redução de custos, o que pode transformar-se em larga vantagem competitiva para uma empresa global em relação aos seus concorrentes. Adicionalmente, as modernas técnicas de administração de estoque têm contribuído para melhoria das taxas de "turnover" que chegam a 100 vezes ao ano, valor muito superior ao que se tinha há alguns anos atrás. A produção automatizada, por sua vez, pode reduzir em dez vezes a necessidade de mão-de-obra. Além disso, o custo de mão-de-obra pode, por exemplo, ser significativamente inferior em uma fábrica na Coréia, quando comparado a países como EUA e Japão.

Consideradas essas "alavancas", uma empresa global tem condições de alcançar excelentes resultados e potencializar sua vantagem competitiva. A produção automatizada e sua intensificação por meio de pesquisas tecnológicas, por exemplo, proporciona elevação da qualidade da produção, o que, combinado com os ganhos de escala, tende a ser irrelevante para os custos e, por outro lado, essencial para as vendas.

Segundo Dyment (1987) as duas principais estratégias param se conseguir vantagem competitiva são: custo e diferencial do produto. O Gráfico 2 ilustra uma aplicação destas estratégias. 
Os desafios administrativos na adoção de sistemas de controle para empresas globalizadas Robson Augusto Dainez Condé, Renato Santiago Quintal, Leonardo Portugal Barcellos, Josir Simeone

Gomes

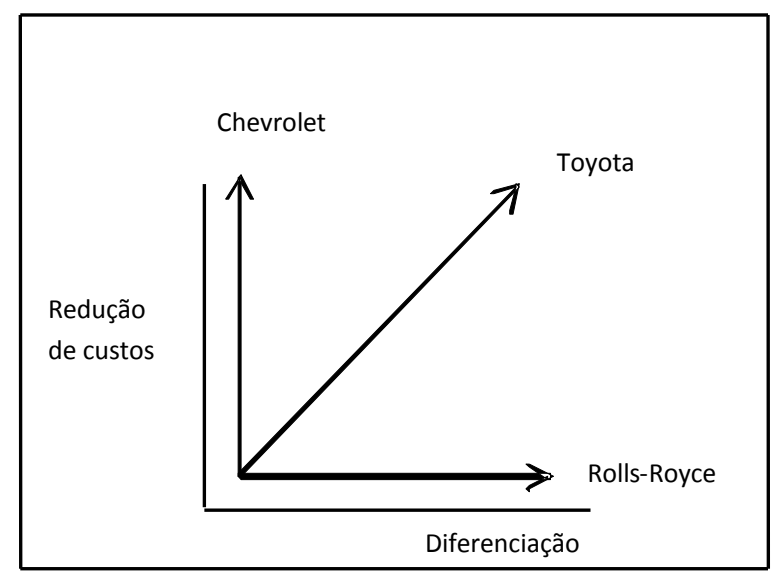

Gráfico 2 Tipos de Estratégias

Fonte: Dyment (1987), p. 23

A Chevrolet, por muitos anos adotou a estratégia de oferecer aos americanos um veículo confiável a custo mais baixo do que os demais carros. A Rolls-Royce, por outro lado, preferiu uma estratégia focada no diferencial do produto, oferecendo um carro de luxo e de qualidade inquestionável, sem se preocupar com o custo. Na década de 1970 a Toyota, com uma estratégia globalizante, lançou um carro que combinava elementos de ambas as estratégias, sendo bem mais barato que o veículo compatível negociado pela Chevrolet, mas contando com alta qualidade. Assim, a Toyota e outros carros fabricados por empresas japonesas tomaram uma significativa fatia do mercado automobilístico americano e mundial.

\subsection{O Sistema de Controle Gerencial da Empresa Globalizada}

Segundo Dyment (1987), um sistema de controle gerencial que atenda às necessidades de uma empresa globalizada deve permitir que a alta administração tenha o controle da estratégia da organização como um todo. Esta estratégia é diferente das utilizadas pelas empresas nacionais e multinacionais, pois a empresa globalizada necessita de uma estratégia de inter-relacionamento de todos os seus recursos.

Uma multinacional, por exemplo, planeja a utilização de seus recursos com base no sucesso de cada unidade de negócio. Para uma empresa globalizada, a avaliação 
Os desafios administrativos na adoção de sistemas de controle para empresas globalizadas

Robson Augusto Dainez Condé, Renato Santiago Quintal, Leonardo Portugal Barcellos, Josir Simeone

Gomes

de uma unidade de negócios quanto à relação retorno-investimento em um determinado local pode ser irrelevante quando considerada a contribuição que este investimento pode trazer para os resultados globais e de longo prazo da organização. Assim, o retorno obtido por uma unidade de um país pode ser negativo e, mesmo assim, novos investimentos podem ser justificados pela contribuição que esta unidade pode trazer para a estratégia mundial.

Portanto, a empresa globalizada deve ter uma administração centralizada e os resultados locais não devem servir como parâmetro para avaliar o sucesso da organização. Os lucros e perdas regionais são importantes para o cálculo dos impostos fiscais e relatórios financeiros, mas não para avaliar o sucesso da administração em atingir suas metas estratégicas (DYMENT, 1987).

A empresa que pretende ampliar suas operações pelo mundo deve identificar suas Posições Estratégicas de Excelência (PEE), ou seja, as capacidades que distinguem a organização e seus produtos quando comparada às demais concorrentes, isto é, as potencialidades que possuem valor significante para o mercado. No Quadro 2 são apresentas algumas PEE's de grandes empresas globalizadas:

\begin{tabular}{|c|c|}
\hline Empresas globalizadas & PEE \\
\hline Gillette & Inovação e qualidade \\
\hline IBM & $\begin{array}{c}\text { Poder financeiro e atendimento ao } \\
\text { consumidor }\end{array}$ \\
\hline Kodak & $\begin{array}{c}\text { Pesquisa/Desenvolvimento e padrão de } \\
\text { qualidade }\end{array}$ \\
\hline L. M. Ericsson & Tecnologia de comando do telefone eletrônico \\
\hline Caterpillar & Serviço e distribuição mundiais \\
\hline McDonald's & Padrão de qualidade mundial \\
\hline
\end{tabular}

Quadro 2: PEE de algumas empresas globais.

Fonte: Dyment (1987), p. 23.

Cada uma, entre as empresas apontadas no Quadro 2, concentrou seus recursos em sua respectiva PEE para atingir posição competitiva no mercado. Segundo Dyment (1987), para atingir o estágio com relativo sucesso, a organização precisa ter bem definidos pontos imprescindíveis como: missão, objetivo, onde pretende chegar, mercado alvo, o escopo do produto ou serviço e, principalmente, a delimitação do 
Os desafios administrativos na adoção de sistemas de controle para empresas globalizadas

Robson Augusto Dainez Condé, Renato Santiago Quintal, Leonardo Portugal Barcellos, Josir Simeone

Gomes

território em que vai operar. Esses elementos nortearão as ações do executivo responsável pela implementação da estratégia da empresa e serão fundamentais ao desempenho da organização.

Além disso, o autor destaca que também precisam ser considerados: (i) os riscos e oportunidades que resultarão das tendências emergentes do mercado; (ii) as mudanças tecnológicas que provavelmente ocorrerão; (iii) os possíveis acontecimentos na economia que afetarão as estratégias da empresa; (iv) o tamanho dos competidores e suas posições estratégicas de excelência; (v) o que a empresa faz bem e que tenha valor para o mercado; e (vi) os recursos que a empresa pode investir para melhorar o seu desempenho global.

Frisa-se que, em seguida, a companhia deverá definir metas para cada uma das PEE. Essas metas devem ser estabelecidas internamente, de modo a apresentar cronologicamente as atividades que devem ser realizadas em direção à posição estratégica de excelência. Para Dyment (1987), a chave para um bom sistema de controle gerencial de uma empresa globalizada está na identificação dos objetivos críticos que precisam ser alcançados prioritariamente.

\subsection{Centralização da Informação}

Em uma empresa globalizada, a matriz administrativa necessita de algumas informações para avaliar se as metas estratégicas estão sendo alcançadas para que, no caso de qualquer desvio eventual, este possa ser corrigido no menor intervalo de tempo possível. Para Dyment (1987), essas informações consistem em:

a) Dados que permitam avaliar o cumprimento de objetivos críticos;

b) Relatórios de controle financeiro que permitam avaliar a viabilidade dos investimentos e a lucratividade da empresa;

c) Obrigações tributárias, legais e administrativas;

d) Informações táticas como, por exemplo, a situação cambial estrangeira, utilizada para resguardar a empresa sobre situações de risco; e

e) A avaliação da gestão organizacional preparada, basicamente, para determinar o premio de remuneração da alta administração. 
Os desafios administrativos na adoção de sistemas de controle para empresas globalizadas

Robson Augusto Dainez Condé, Renato Santiago Quintal, Leonardo Portugal Barcellos, Josir Simeone

Gomes

Ainda de acordo com o autor, os relatórios financeiros da empresa globalizada tendem a ser menos extensos do que os elaborados para empresas multinacionais, sendo que o controle financeiro deve atender às seguintes exigências específicas:

a) As contas a receber e estoques devem ser controlados no curto prazo;

b) As alterações no ativo imobilizado devem ser controladas através de relatórios que avaliem os gastos de capital;

c) As obrigações correntes devem ser administradas como parte dos controles gerenciais de caixa;

d) Os empréstimos devem ser de curto prazo e em países com o custo mais baixo, levando-se em conta o risco cambial e as restrições ao controle da moeda;

e) As alterações nos débitos de longo prazo e no capital integralizado devem ser resultado de decisões criteriosas, relatadas sempre que ocorrerem.

Os relatórios mensais tradicionais referentes aos balanços, demonstrações de resultados e a variedade de tabelas de apoio usadas em muitas das empresas multinacionais fornecem muito pouco controle adicional e não se justificam para a maioria das empresas globalizadas.

Os planos de bonificação de gerentes, largamente estudados na área financeira, podem ser compostos de: (i) salário básico, independente de resultados; (ii) participação nos lucros; e (iii) um prêmio pela atuação com base em metas alcançadas e em resultados positivos obtidos nas avaliações gerenciais

O objetivo dos controles gerenciais em uma empresa globalizada é comprovar que as estratégias mundiais da organização estão sendo implementadas com sucesso. Assim, a principal função dos relatórios gerenciais é revelar se os objetivos críticos atribuídos a cada unidade estão sendo atingidos. Caso os relatórios mostrem que algum objetivo não foi alcançado, deve-se atuar na correção desse desvio, sendo que, quando um objetivo não é atingido por uma situação alheia ao controle da administração, a própria estratégia global deve ser revista e corrigida (DYMENT, 1987).

Assim, os relatórios de progresso sobre o cumprimento de metas não devem ser muito extensos ou detalhados. Da mesma forma, o sistema de informação sobre 
Os desafios administrativos na adoção de sistemas de controle para empresas globalizadas

Robson Augusto Dainez Condé, Renato Santiago Quintal, Leonardo Portugal Barcellos, Josir Simeone

Gomes

alterações cambiais deve ser exigido em casos específicos, para manutenção do controle de risco sobre o cambio, bem como o capital de giro deve ser obtido a taxas de financiamento mais baixas, que não comprometam a situação cambial da organização.

\section{CONSIDERAÇÕES FINAIS}

A gestão de empresas globalizadas desponta como uma nova perspectiva nos estudos organizacionais e sugere alguns cuidados, os quais, conforme discutido ao longo do presente estudo, podem ser cruciais para a sobrevivência das organizações.

O estudo deste tema tem alcançado relevância nos últimos anos, principalmente em virtude das constantes modificações impostas pela globalização dos mercados, o que tem como consequência imediata a homogeneização da demanda.

Muitos empresários brasileiros estão encontrando na globalização uma oportunidade de precaução contra crises econômicas, encontrando na diversidade de mercados consumidores e fornecedores o segredo para manter negócios sempre rentáveis. Assim, o presente estudo é relevante para os empresários que pretendem aumentar suas fronteiras de atuação e para aqueles que desejam mitigar os riscos de seus negócios.

Assim, tais empresários devem estar atentos à visão trazida pela literatura acadêmica, que pode ser brevemente sintetizada da seguinte forma: empresas podem se estabelecer como nacionais, multinacionais ou globalizadas e podem migrar de um modelo para outro, mas, acompanhando as mudanças no interior dessa tipologia, o sistema de controle gerencial deve ser modificado de modo a estar adequado a uma nova estratégia da unidade de negócios.

Desta forma, a decisão de disseminar suas atividades para outros países deve ser fundamentada em um estudo aprofundado sobre as implicações de tal decisão. A opção por uma estratégia globalizante pode ser determinante para que a empresa obtenha vantagem competitiva em relação à concorrência e, nesse contexto, a adoção de um sistema de controle apropriado pode ser a chave para potencializar a capacidade competitiva da organização. Os riscos envolvidos na manutenção das modalidades de 
Os desafios administrativos na adoção de sistemas de controle para empresas globalizadas

Robson Augusto Dainez Condé, Renato Santiago Quintal, Leonardo Portugal Barcellos, Josir Simeone

controles anteriores podem representar o fracasso da empreitada rumo à expansão e/ou diversificação desejadas.

\section{REFERÊNCIAS}

ANTHONY, R. N.; GOVINDARAJAN, V. (2002). Sistemas de controle gerencial. São Paulo: Atlas.

BARTLETT, C. A.; GHOSHAL, S. (1992). Gerenciando empresas no exterior: a solução transnacional. São Paulo: Makron Books.

CANALS, J. (1994). La internacionalizacion de la empresa. Madrid: McGraw-Hill.

DYMENT, John J. (1987). Strategies and management controls for global corporations. The journal of business strategy, v.7, n.4, spring. P.20-6.

FERNANDES, A. F. Neto. (2006). A Importância da Globalização para as Empresas Brasileiras. THESIS, São Paulo, ano III, v .5, p. 1-20, 1ํ Semestre.

FLEURY, Maria Tereza Leme (Org). (2007). Internacionalização e os Países Emergentes. São Paulo: Atlas.

GOMES, J. S, OLIVEIRA, R. R. (2011). Inserção do Tema Internacionalização de Empresas em Artigos Produzidos no Programa de Mestrado em Ciências Contábeis da UERJ. VII Congresso Nacional de Excelência em Gestão.

GOMES, J. S., RODRIGUES, A. (2005). Um Estudo Exploratório Sobre Controle Gerencial em Empresas Brasileiras Internacionalizadas do Setor de Serviços: Quatro Estudos de Casos. In: XXIX Enanpad.

MAZO, M. S., TEIXEIRA, M. C., HERNANDES C. A. Estratégia e Globalização. VII SEMEAD. Disponível em: <http://www.ead.fea.usp.br/Semead/7semead/paginas/artigos\%20recebidos/Comercio\% 20exterior/COMEX12-_Estrat\%E9gia_e_Globaliza\%E7\%E3o.PDF>. Acesso em: 20/set/2011.

MERCHANT, K. (1998). Modern management control systems: text \& cases. Upper Saddle River: Prentice-Hall.

OTLEY, David. (1994). Management control in contemporary organizations: towards a wider framework. Management Accounting Research, n.5, p. 289-299. 
Os desafios administrativos na adoção de sistemas de controle para empresas globalizadas Robson Augusto Dainez Condé, Renato Santiago Quintal, Leonardo Portugal Barcellos, Josir Simeone

ROCHA, Angela da. (2004). O constructo da distância psicológica: componentes, mediadores e assimetria. In: Hemais, Carlos A. (Org.). O Desafio dos mercados externos: Teoria e Prática na internacionalização da firma. v. 1. Rio de Janeiro: Mauad.

ROCHA, Angela da; SILVA, Jorge Ferreira da; CARNEIRO, Jorge. (2007). Expansão internacional das empresas brasileiras: expansão e síntese. In: FLEURY, Afonso; FLEURY, Maria Tereza Leme (Orgs.). Internacionalização e os países emergentes. São Paulo: Atlas.

URBASCH, Gerhard. (2004). A Globalização Brasileira: A conquista dos mercados mundiais por empresas nacionais. Rio de Janeiro: Elsevier.

Data de Submissão: 05/01/2012

Data de Aceite: 26/03/2012 